Adv. Appl. Math. 136 (2022), Article ID 102319.

\title{
ON MOTZKIN NUMBERS AND CENTRAL TRINOMIAL COEFFICIENTS
}

\author{
ZHI-WEI SUN \\ Department of Mathematics, Nanjing University \\ Nanjing 210093, People's Republic of China \\ zwsun@nju.edu.cn \\ http://maths.nju.edu.cn/ zwsun
}

\begin{abstract}
$$
\begin{gathered}
\frac{2}{n} \sum_{k=1}^{n}(2 k+1) M_{k}^{2} \in \mathbb{Z}, \\
\frac{n^{2}\left(n^{2}-1\right)}{6} \mid \sum_{k=0}^{n-1} k(k+1)(8 k+9) T_{k} T_{k+1},
\end{gathered}
$$
\end{abstract}
ABstract. The Motzkin numbers $M_{n}=\sum_{k=0}^{n}\left(\begin{array}{c}n \\ 2 k\end{array}\right)\left(\begin{array}{c}2 k \\ k\end{array}\right) /(k+1)(n=0,1,2, \ldots)$ and the central trinomial coefficients $T_{n}(n=0,1,2, \ldots)$ given by the constant term of $\left(1+x+x^{-1}\right)^{n}$, have many combinatorial interpretations. In this paper we establish the following surprising arithmetic properties of them with $n$ any positive integer:

and also

$$
\sum_{k=0}^{n-1}(k+1)(k+2)(2 k+3) M_{k}^{2} 3^{n-1-k}=n(n+1)(n+2) M_{n} M_{n-1}
$$

\section{INTRODUCTION}

In combinatorics, the Motzkin number $M_{n}$ with $n \in \mathbb{N}=\{0,1,2, \ldots\}$ is the number of lattice paths from the point $(0,0)$ to the point $(n, 0)$ which never dip below the line $y=0$ and are made up only of the allowed steps $(1,0)$ (east), $(1,1)$ (northeast) and $(1,-1)$ (southeast). It is well known that

$$
M_{n}=\sum_{k=0}^{\lfloor n / 2\rfloor}\left(\begin{array}{c}
n \\
2 k
\end{array}\right) C_{k}
$$

where $C_{k}$ denotes the Catalan number $\left(\begin{array}{c}2 k \\ k\end{array}\right)-\left(\begin{array}{c}2 k \\ k+1\end{array}\right)=\left(\begin{array}{c}2 k \\ k\end{array}\right) /(k+1)$.

2020 Mathematics Subject Classification. Primary 05A10, 05A19; Secondary 11A07, 11B75.

Keywords. Motzkin number, central trinomial coefficient, Delannoy number, Schröder number, congruence.

Supported by the National Natural Science Foundation of China (grant 11971222). 
For $n \in \mathbb{N}$, the central trinomial coefficient $T_{n}$ is the constant term in the expansion of $\left(1+x+x^{-1}\right)^{n}$. By the multi-nomial theorem, we see that

$$
T_{n}=\sum_{k=0}^{\lfloor n / 2\rfloor}\left(\begin{array}{c}
n \\
2 k
\end{array}\right)\left(\begin{array}{c}
2 k \\
k
\end{array}\right)=\sum_{k=0}^{n}\left(\begin{array}{l}
n \\
k
\end{array}\right)\left(\begin{array}{c}
n-k \\
k
\end{array}\right)
$$

It is known that $T_{n}$ coincides with the number of lattice paths from the point $(0,0)$ to $(n, 0)$ with only allowed steps $(1,0)$ (east), $(1,1)$ (northeast) and $(1,-1)$ (southeast).

The Motzkin numbers, the Catalan numbers and the central trinomial coefficients arise naturally in enumerative combinatorics. As the Fibonacci numbers arising from combinatorics have rich number-theoretic properties, we think that important combinatorial quantities like $M_{n}$ and $T_{n}$ with $n \in \mathbb{N}$ should also have nice arithmetic properties. For example, in [S14a] we conjectured that for any $n \in \mathbb{Z}^{+}=\{1,2,3, \ldots\}$ the arithmetic mean of the $n$ numbers $(8 k+5) T_{k}^{2}(k=$ $0, \ldots, n-1)$ is always an integer, and this was later confirmed by Y.-P. Mu and the author [MS] via symbolic computation. Motivated by congruence properties of such numbers, we found in [S14b, S20] many series for $1 / \pi$ involving central trinomial coefficients or their extensions. For example, in [S20, Section 10] we conjectured the combinatorial identity

$$
\sum_{k=1}^{\infty} \frac{(105 k-44) T_{k-1}}{k^{2}\left(\begin{array}{c}
2 k \\
k
\end{array}\right)^{2} 3^{k-1}}=\frac{5 \pi}{\sqrt{3}}+6 \log 3
$$

based on the conjectural congruence

$$
p^{2} \sum_{k=1}^{p-1} \frac{(105 k-44) T_{k-1}}{k^{2}\left(\begin{array}{c}
2 k \\
k
\end{array}\right)^{2} 3^{k-1}} \equiv 11\left(\frac{p}{3}\right)+\frac{p}{2}\left(13-35\left(\frac{p}{3}\right)\right) \quad\left(\bmod p^{2}\right),
$$

where $p$ is a prime greater than 3 and $(-)$ is the Legendre symbol. Thus it is interesting to investigate congruence properties of combinatorial quantities like $M_{n}$ and $T_{n}$ with $n \in \mathbb{N}$, and the study in turn may stimulate us to find some new combinatorial identities.

Let $p>3$ be a prime. In [S14a, Conjecture 1.1(ii)] we conjectured

$$
\sum_{k=0}^{p-1} M_{k}^{2} \equiv(2-6 p)\left(\frac{p}{3}\right) \quad\left(\bmod p^{2}\right), \quad \sum_{k=0}^{p-1} k M_{k}^{2} \equiv(9 p-1)\left(\frac{p}{3}\right) \quad\left(\bmod p^{2}\right),
$$

and

$$
\sum_{k=0}^{p-1} T_{k} M_{k} \equiv \frac{4}{3}\left(\frac{p}{3}\right)+\frac{p}{6}\left(1-9\left(\frac{p}{3}\right)\right) \quad\left(\bmod p^{2}\right) .
$$

The three supercongruences look curious and challenging.

Motivated by the above conjectures, we establish the following new results. 
Theorem 1.1. (i) For any $n \in \mathbb{Z}^{+}$, we have

$$
s(n):=\frac{2}{n} \sum_{k=1}^{n}(2 k+1) M_{k}^{2} \in \mathbb{Z}
$$

(ii) For any prime $p>3$, we have

$$
\sum_{k=0}^{p-1}(2 k+1) M_{k}^{2} \equiv 12 p\left(\frac{p}{3}\right) \quad\left(\bmod p^{2}\right)
$$

Remark 1.1. The values of $s(1), \ldots, s(10)$ are as follows:

6, 23, 90, 432, 2286, 13176, 80418, 513764, 3400518, 23167311.

Theorem 1.2. For any integer $n \geqslant 2$, we have

$$
\frac{n^{2}\left(n^{2}-1\right)}{6} \mid \sum_{k=0}^{n-1} k(k+1)(8 k+9) T_{k} T_{k+1}
$$

Remark 1.2. If we define

$$
t(n):=\frac{6}{n^{2}\left(n^{2}-1\right)} \sum_{k=0}^{n-1} k(k+1)(8 k+9) T_{k} T_{k+1} \quad(n=2,3, \ldots),
$$

then the values of $t(2), t(3), \ldots, t(10)$ are as follows:

51, 271, 1398, 8505, 54387, 367551, 2570931, 18510739, 136282347.

Let $b, c \in \mathbb{Z}$ and $n \in \mathbb{N}$. The generalized central trinomial coefficient $T_{n}(b, c)$ denotes the coefficient of $x^{n}$ in the expansion of $\left(x^{2}+b x+c\right)^{n}$ (cf. [S14a] and $[\mathrm{S} 14 \mathrm{~b}])$. By the multi-nomial theorem, we see that

$$
T_{n}(b, c)=\sum_{k=0}^{\lfloor n / 2\rfloor}\left(\begin{array}{c}
n \\
2 k
\end{array}\right)\left(\begin{array}{c}
2 k \\
k
\end{array}\right) b^{n-2 k} c^{k}
$$

The generalized Motzkin number $M_{n}(b, c)$ introduced in [S14a] is given by

$$
M_{n}(b, c)=\sum_{k=0}^{\lfloor n / 2\rfloor}\left(\begin{array}{c}
n \\
2 k
\end{array}\right) C_{k} b^{n-2 k} c^{k}
$$


Note that $T_{n}(1,1)=T_{n}, M_{n}(1,1)=M_{n}, T_{n}(2,1)=\left(\begin{array}{c}2 n \\ n\end{array}\right)$ and $M_{n}(2,1)=C_{n+1}$. Also, $T_{n}(3,2)$ coincides with the (central) Delannoy number

$$
D_{n}=\sum_{k=0}^{n}\left(\begin{array}{l}
n \\
k
\end{array}\right)\left(\begin{array}{c}
n+k \\
k
\end{array}\right)=\sum_{k=0}^{n}\left(\begin{array}{c}
n+k \\
2 k
\end{array}\right)\left(\begin{array}{c}
2 k \\
k
\end{array}\right),
$$

which counts lattice paths from $(0,0)$ to $(n, n)$ in which only east $(1,0)$, north $(0,1)$, and northeast $(1,1)$ steps are allowed (cf. R. P. Stanley [St99, p. 185]). And $M_{n}(3,2)$ equals the little Schröder number

$$
s_{n+1}=\sum_{k=1}^{n+1} N(n+1, k) 2^{n+1-k}
$$

with the Narayana number $N(m, k)(m \geqslant k \geqslant 1)$ given by

$$
N(m, k):=\frac{1}{m}\left(\begin{array}{c}
m \\
k
\end{array}\right)\left(\begin{array}{c}
m \\
k-1
\end{array}\right) \in \mathbb{Z} .
$$

The little Schröder numbers and the Narayana numbers also have many combinatorial interpretations (cf. [St97] and [Gr, pp. 268-281]). See also [S11, S18b] for some congruences involving the Delannoy numbers or the little Schröder numbers.

Theorem 1.3. Let $b, c \in \mathbb{Z}$ with $b \neq 0$ and $d=b^{2}-4 c \neq 0$, and let $n \in \mathbb{Z}^{+}$. Then

$$
b \frac{n(n+1)}{2} \mid \sum_{k=1}^{n} k T_{k}(b, c) T_{k-1}(b, c) d^{n-k}
$$

and

$$
b \frac{n^{2}(n+1)^{2}}{4} \mid 3 \sum_{k=1}^{n} k^{3} T_{k}(b, c) T_{k-1}(b, c) d^{n-k} .
$$

Also,

$$
\frac{(2, n)}{n(n+1)(n+2)} \sum_{k=0}^{n-1}(k+1)(k+2)(2 k+3) M_{k}(b, c)^{2} d^{n-1-k} \in \mathbb{Z}
$$

and

$$
\sum_{k=0}^{n-1} \frac{(k+1)(k+2)(2 k+3)}{n(n+1)(n+2)} M_{k}(b, c)^{2}(-d)^{n-1-k}=\frac{M_{n}(b, c) M_{n-1}(b, c)}{b} \in \mathbb{Z},
$$

where $(m, n)$ denotes the greatest common divisor of two integers $m$ and $n$.

Remark 1.3. For each $n \in \mathbb{Z}^{+}$, (1.7) with $b=c=1$ gives the curious identity

$$
\sum_{k=0}^{n-1}(k+1)(k+2)(2 k+3) M_{k}^{2} 3^{n-1-k}=n(n+1)(n+2) M_{n} M_{n-1} .
$$

In the case $b=3$ and $c=2$, Theorem 1.3 yields the following consequence. 
Corollary 1.1. For any $n \in \mathbb{Z}^{+}$we have

$$
\begin{gathered}
3 \frac{n(n+1)}{2}\left|\sum_{k=1}^{n} k D_{k} D_{k-1}, \frac{n^{2}(n+1)^{2}}{4}\right| \sum_{k=1}^{n} k^{3} D_{k} D_{k-1}, \\
\frac{n(n+1)(n+2)}{(2, n)} \mid \sum_{k=1}^{n} k(k+1)(2 k+1) s_{k}^{2},
\end{gathered}
$$

and

$$
\frac{1}{n(n+1)(n+2)} \sum_{k=1}^{n} k(k+1)(2 k+1)(-1)^{n-k} s_{k}^{2}=\frac{s_{n} s_{n+1}}{3} \in \mathbb{Z}
$$

Theorems 1.1-1.3 are quite sophisticated and their proofs need various techniques. We will prove Theorems 1.1-1.3 in Sections 2-4 respectively. In Section 5 we are going to pose some related conjectures for further research.

\section{Proof of Theorem 1.1}

For $n \in \mathbb{Z}^{+}$, in $[\mathrm{S} 18 \mathrm{~b}]$ we introduced the polynomial

$$
s_{n}(x):=\sum_{k=1}^{n} N(n, k) x^{k-1}(x+1)^{n-k}
$$

for which $s_{n}(1)$ is just the little Schróder number $s_{n}$. For $n \in \mathbb{N}$, define

$$
S_{n}(x)=\sum_{k=0}^{n}\left(\begin{array}{l}
n \\
k
\end{array}\right)\left(\begin{array}{c}
n+k \\
k
\end{array}\right) \frac{x^{k}}{k+1}=\sum_{k=0}^{n}\left(\begin{array}{c}
n+k \\
2 k
\end{array}\right) C_{k} x^{k} .
$$

Then $S_{n}(1)$ equals the large Schröder number $S_{n}$ which counts the lattice paths from the point $(0,0)$ to $(n, n)$ with steps $(1,0),(0,1)$ and $(1,1)$ that never rise above the line $y=x$. As proved in [S18b], we have

$$
S_{n}(x)=(x+1) s_{n}(x) \quad \text { for all } n \in \mathbb{Z}^{+} .
$$

Lemma 2.1. (i) For any $n \in \mathbb{Z}^{+}$we have

$$
n(n+1) s_{n}(x)^{2}=\sum_{k=1}^{n}\left(\begin{array}{c}
n+k \\
2 k
\end{array}\right)\left(\begin{array}{c}
2 k \\
k
\end{array}\right)\left(\begin{array}{c}
2 k \\
k+1
\end{array}\right)(x(x+1))^{k-1} .
$$

(ii) Let $b, c \in \mathbb{Z}$ with $d=b^{2}-4 c \neq 0$. For any $n \in \mathbb{N}$ we have

$$
M_{n}(b, c)=(\sqrt{d})^{n} s_{n+1}\left(\frac{b / \sqrt{d}-1}{2}\right) .
$$


Proof. As $(x+1) s_{n}(x)=S_{n}(x)$ by (2.3), the identity (2.4) has the equivalent version

$$
n(n+1) S_{n}(x)^{2}=\sum_{k=1}^{n}\left(\begin{array}{c}
n+k \\
2 k
\end{array}\right)\left(\begin{array}{c}
2 k \\
k
\end{array}\right)\left(\begin{array}{c}
2 k \\
k+1
\end{array}\right) x^{k-1}(x+1)^{k+1}
$$

which appeared as [S12a, (2.1)]. So (2.4) holds. The identity (2.5) was proved in [S18b, Lemma 3.1].

Remark 2.1. For $n \in \mathbb{N}$ and $b, c \in \mathbb{Z}$ with $b^{2} \neq 4 c$, by combining the two parts of Lemma 2.1 we obtain that

$$
M_{n}(b, c)^{2}=\frac{1}{(n+1)(n+2)} \sum_{k=1}^{n+1}\left(\begin{array}{c}
n+k+1 \\
2 k
\end{array}\right)\left(\begin{array}{c}
2 k \\
k
\end{array}\right)\left(\begin{array}{c}
2 k \\
k+1
\end{array}\right) c^{k-1}\left(b^{2}-4 c\right)^{n+1-k} .
$$

Lemma 2.2. For any $n \in \mathbb{Z}^{+}$we have

$$
\begin{aligned}
& \sum_{k=1}^{n}(2 k+1) M_{k}^{2} \\
= & \sum_{k=0}^{n+1} \frac{(4 n-2 k+3)(n+k+2)}{n+2}\left(\begin{array}{c}
n+k+1 \\
2 k
\end{array}\right)\left(\begin{array}{c}
2 k \\
k
\end{array}\right)\left(\begin{array}{c}
2 k+1 \\
k
\end{array}\right)(-3)^{n+1-k} .
\end{aligned}
$$

Proof. In view of (2.6), we have

$$
\begin{aligned}
\sum_{k=0}^{n}(2 k+1) M_{k}^{2} & =\sum_{k=0}^{n} \frac{2 k+1}{(k+1)(k+2)} \sum_{j=1}^{k+1}\left(\begin{array}{c}
k+j+1 \\
2 j
\end{array}\right)\left(\begin{array}{c}
2 j \\
j
\end{array}\right)\left(\begin{array}{c}
2 j \\
j+1
\end{array}\right)(-3)^{k+1-j} \\
& =\sum_{k=0}^{n} \frac{2 k+1}{(k+1)(k+2)} \sum_{l=0}^{k}\left(\begin{array}{c}
k+l+2 \\
2 l+2
\end{array}\right)\left(\begin{array}{c}
2 l+2 \\
l+1
\end{array}\right)\left(\begin{array}{c}
2 l+2 \\
l
\end{array}\right)(-3)^{k-l} \\
& =\sum_{k=0}^{n} \sum_{l=0}^{n} F(k, l),
\end{aligned}
$$

where

$$
F(k, l):=\frac{2 k+1}{(k+1)(k+2)}\left(\begin{array}{c}
k+l+2 \\
2 l+2
\end{array}\right)\left(\begin{array}{c}
2 l+2 \\
l+1
\end{array}\right)\left(\begin{array}{c}
2 l+2 \\
l
\end{array}\right)(-3)^{k-l} .
$$

By the telescoping method developed by Chen, Hou and $\mathrm{Mu}[\mathrm{CHM}]$ and applied by $\mathrm{Mu}$ and Sun $[\mathrm{MS}]$, the double sum can be reduced to a single sum:

$$
\sum_{k=0}^{n} \sum_{l=0}^{n} F(k, l)=1+(4 n+3)(-3)^{n+1}+\sum_{j=0}^{n}(-3)^{n-j} \frac{(4 n-2 j+1)(n+j+3) !(2 j+3) !}{(n+2)(n-j) !(j+2)(j+1) !^{4}} .
$$


Therefore

$$
\begin{aligned}
& \sum_{k=1}^{n}(2 k+1) M_{k}^{2} \\
= & \sum_{j=-1}^{n}(-3)^{n-j} \frac{(4 n-2 j+1)(n+j+3) !(2 j+3) !}{(n+2)(n-j) !(j+2)(j+1) !^{4}} \\
= & \sum_{k=0}^{n+1}(-3)^{n+1-k} \frac{(4 n-2 k+3)(n+k+2) !(2 k+1) !}{(n+2)(n+1-k) !(k+1) k !^{4}} \\
= & \sum_{k=0}^{n+1} \frac{(4 n-2 k+3)(n+k+2)}{n+2}\left(\begin{array}{c}
n+k+1 \\
2 k
\end{array}\right)\left(\begin{array}{c}
2 k \\
k
\end{array}\right)\left(\begin{array}{c}
2 k+1 \\
k
\end{array}\right)(-3)^{n+1-k}
\end{aligned}
$$

and this concludes the proof.

For each integer $n$ we set

$$
[n]_{q}=\frac{1-q^{n}}{1-q}
$$

which is the usual $q$-analogue of $n$. For any $n \in \mathbb{Z}$, we define

$$
\left[\begin{array}{l}
n \\
0
\end{array}\right]_{q}=1 \text { and }\left[\begin{array}{l}
n \\
k
\end{array}\right]_{q}=\frac{\prod_{j=0}^{k-1}[n-j]_{q}}{\prod_{j=1}^{k}[j]_{q}} \text { for } k=1,2,3, \ldots
$$

Obviously $\lim _{q \rightarrow 1}\left[\begin{array}{l}n \\ k\end{array}\right]_{q}=\left(\begin{array}{l}n \\ k\end{array}\right)$ for all $k \in \mathbb{N}$ and $n \in \mathbb{Z}$. It is easy to see that

$$
\left[\begin{array}{l}
n \\
k
\end{array}\right]_{q}=q^{k}\left[\begin{array}{c}
n-1 \\
k
\end{array}\right]_{q}+\left[\begin{array}{l}
n-1 \\
k-1
\end{array}\right]_{q} \quad \text { for all } k, n=1,2,3, \ldots
$$

By this recursion, $\left[\begin{array}{l}n \\ k\end{array}\right]_{q} \in \mathbb{Z}[q]$ for all $k, n \in \mathbb{N}$. For any integers $a, b$ and $n>0$, clearly

$$
a \equiv b \quad(\bmod n) \Longrightarrow[a]_{q} \equiv[b]_{q} \quad\left(\bmod [n]_{q}\right)
$$

Let $n$ be a positive integer. The cyclotomic polynomial

$$
\Phi_{n}(q):=\prod_{\substack{a=1 \\(a, n)=1}}^{n}\left(q-e^{2 \pi i a / n}\right) \in \mathbb{Z}[q]
$$

is irreducible in the ring $\mathbb{Z}[q]$. It is well-known that

$$
q^{n}-1=\prod_{d \mid n} \Phi_{d}(q)
$$

Note that $\Phi_{1}(q)=q-1$. 
Lemma 2.3. For any $a, b \in \mathbb{N}$ and $n \in \mathbb{Z}^{+}$, we have

$$
\sum_{k=0}^{n-1}\left[\begin{array}{c}
n+1 \\
k
\end{array}\right]_{q}^{a}\left[\begin{array}{c}
n+k \\
k
\end{array}\right]_{q}^{b}\left[\begin{array}{c}
2 k \\
k
\end{array}\right]_{q}[k+2]_{q}\left(-[3]_{q}\right)^{n-1-k} \equiv 0 \quad\left(\bmod [n]_{q}\right)
$$

Proof. (2.9) is trivial in the case $n=1$. Below we assume $n>1$. As

$$
[n]_{q}=\prod_{1<d \mid n} \Phi_{d}(q)
$$

and $\Phi_{2}(q), \Phi_{3}(q), \ldots$ are pairwise coprime, it suffices to show that the sum in $(2.9)$ is divisible by $\Phi_{d}(q)$ for any given divisor $d>1$ of $n$.

A well-known $q$-Lucas theorem (see, e.g., [O]) states that if $a, b, d, s, t \in \mathbb{N}$ with $s<d$ and $t<d$ then

$$
\left[\begin{array}{l}
a d+s \\
b d+t
\end{array}\right]_{q} \equiv\left(\begin{array}{l}
a \\
b
\end{array}\right)\left[\begin{array}{l}
s \\
t
\end{array}\right]_{q} \quad\left(\bmod \Phi_{d}(q)\right)
$$

Let $S$ denote the sum in (2.9) and write $n=d m$ with $m \in \mathbb{Z}^{+}$. Then

$$
\begin{aligned}
& S=\sum_{j=0}^{m-1} \sum_{r=0}^{d-1}\left[\begin{array}{c}
m d+1 \\
j d+r
\end{array}\right]_{q}^{a}\left[\begin{array}{c}
m d+j d+r \\
j d+r
\end{array}\right]_{q}^{b}\left[\begin{array}{c}
2 j d+2 r \\
j d+r
\end{array}\right]_{q}[j d+r+2]_{q}\left(-[3]_{q}\right)^{m d-1-(j d+r)} \\
& \equiv \sum_{j=0}^{m-1} \sum_{r=0}^{d-1}\left(\begin{array}{c}
m \\
j
\end{array}\right)^{a}\left[\begin{array}{c}
1 \\
r
\end{array}\right]_{q}^{a}\left(\begin{array}{c}
m+j \\
j
\end{array}\right)^{b}\left[\begin{array}{l}
r \\
r
\end{array}\right]_{q}^{b}\left[\begin{array}{c}
2 j d+2 r \\
j d+r
\end{array}\right]_{q}[r+2]_{q}\left(-[3]_{q}\right)^{(m-j) d-(r+1)} \\
& \equiv \sum_{j=0}^{m-1}\left(\begin{array}{c}
m \\
j
\end{array}\right)^{a}\left(\begin{array}{c}
m+j \\
j
\end{array}\right)^{b} \sum_{r=0}^{1}\left[\begin{array}{c}
2 j d+2 r \\
j d+r
\end{array}\right]_{q}[r+2]_{q}\left(-[3]_{q}\right)^{(m-j) d-(r+1)} \\
& \equiv \sum_{j=0}^{m-1}\left(\begin{array}{c}
m \\
j
\end{array}\right)^{a}\left(\begin{array}{c}
m+j \\
j
\end{array}\right)^{b}\left(\begin{array}{c}
2 j \\
j
\end{array}\right)\left[\begin{array}{l}
0 \\
0
\end{array}\right]_{q}[2]_{q}\left(-[3]_{q}\right)^{(m-j) d-1}
\end{aligned}
$$

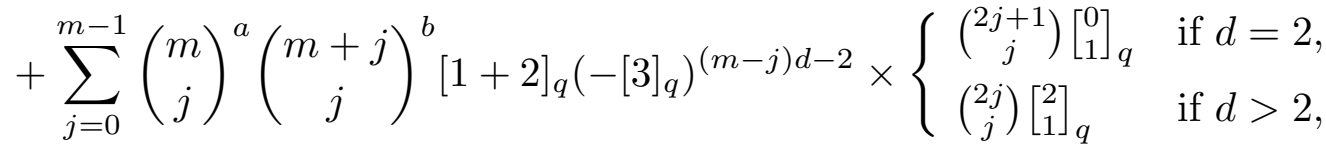

$$
\begin{aligned}
& \equiv 0\left(\bmod \Phi_{d}(q)\right) \text {. }
\end{aligned}
$$

(Note that $[2]_{q}=1+q=\Phi_{2}(q)$.) This concludes the proof.

Lemma 2.4. For any prime $p>3$ we have

$$
\sum_{k=1}^{p-1} \frac{\left(\begin{array}{c}
2 k \\
k
\end{array}\right)}{k 3^{k}} \equiv \frac{3^{p-1}-1}{p} \quad(\bmod p)
$$


Proof. Let $u_{n}=\left(\frac{n}{3}\right)$ for $n \in \mathbb{N}$. Then $u_{0}=0, u_{1}=1$ and $u_{n+1}=-u_{n}-u_{n-1}$ for all $n=1,2,3, \ldots$ Applying [S12b, Lemma 3.5] with $m=1$, we obtain

$$
\sum_{k=1}^{p-1} \frac{\left(\begin{array}{c}
2 k \\
k
\end{array}\right)}{k 3^{k}} \equiv \frac{(-3)^{p-1}-1}{p}-\frac{1}{2}\left(\frac{-3}{p}\right) \frac{u_{p-\left(\frac{-3}{p}\right)}}{p}(\bmod p)
$$

Note that $u_{p-\left(\frac{-3}{p}\right)}=0$ since $p \equiv\left(\frac{-3}{p}\right)(\bmod 3)$. So $(2.10)$ holds.

Proof of Theorem 1.1. (i) Observe that

$$
\frac{4}{n+2} \equiv \begin{cases}4 / 2=2(\bmod n) & \text { if } 2 \nmid n, \\ 2 /(n / 2+1) \equiv 2(\bmod n) & \text { if } 2 \mid n .\end{cases}
$$

Thus, for each $k \in\{1, \ldots, n+1\}$, we have

$$
2 \times \frac{\left(\begin{array}{c}
2 k \\
k
\end{array}\right)}{n+2}=\frac{4}{n+2}\left(\begin{array}{c}
2 k-1 \\
k
\end{array}\right) \equiv 2\left(\begin{array}{c}
2 k-1 \\
k
\end{array}\right)=\left(\begin{array}{c}
2 k \\
k
\end{array}\right) \quad(\bmod n) .
$$

Combining this with (2.7) we see that

$$
\begin{aligned}
& 2 \sum_{k=1}^{n}(2 k+1) M_{k}^{2} \\
\equiv & 2(4 n+3)(-3)^{n+1} \\
& +\sum_{k=1}^{n+1}(4 n-2 k+3)(n+k+2)\left(\begin{array}{c}
n+k+1 \\
2 k
\end{array}\right)\left(\begin{array}{c}
2 k \\
k
\end{array}\right)\left(\begin{array}{c}
2 k+1 \\
k
\end{array}\right)(-3)^{n+1-k} \\
\equiv & -\sum_{k=0}^{n+1}(2 k-3)(k+2)\left(\begin{array}{c}
n+k+1 \\
n+1
\end{array}\right)\left(\begin{array}{c}
n+1 \\
k
\end{array}\right)\left(\begin{array}{c}
2 k+1 \\
k
\end{array}\right)(-3)^{n+1-k} \\
\equiv & -\sum_{k=0}^{n+1}(2 k-3)(k+2) \frac{n+k+1}{n+1}\left(\begin{array}{c}
n+k \\
k
\end{array}\right)\left(\begin{array}{c}
n+1 \\
k
\end{array}\right)(2 k+1) C_{k}(-3)^{n+1-k} \\
\equiv & -\sum_{k=0}^{n+1}(2 k-3)(k+2)(k+1)\left(\begin{array}{c}
n+k \\
k
\end{array}\right)\left(\begin{array}{c}
n+1 \\
k
\end{array}\right)(2 k+1) C_{k}(-3)^{n+1-k}(\bmod n) .
\end{aligned}
$$

For each $k=0, \ldots, n+1$, clearly

$$
k(k-1)\left(\begin{array}{c}
n+1 \\
k
\end{array}\right)=n(n+1)\left(\begin{array}{c}
n-1 \\
n+1-k
\end{array}\right) \equiv 0 \quad(\bmod n) .
$$

Since $(2 k-3)(2 k+1)=4 k(k-1)-3$, by the above we have

$$
2 \sum_{k=1}^{n}(2 k+1) M_{k}^{2} \equiv-\sum_{k=0}^{n+1}\left(\begin{array}{c}
n+1 \\
k
\end{array}\right)\left(\begin{array}{c}
n+k \\
k
\end{array}\right)\left(\begin{array}{c}
2 k \\
k
\end{array}\right)(k+2)(-3)^{n+2-k} \quad(\bmod n)
$$


Note that

$$
\begin{aligned}
& \sum_{k=n}^{n+1}\left(\begin{array}{c}
n+1 \\
k
\end{array}\right)\left(\begin{array}{c}
n+k \\
k
\end{array}\right)\left(\begin{array}{c}
2 k \\
k
\end{array}\right)(k+2)(-3)^{n+2-k} \\
= & \left(\begin{array}{c}
n+1 \\
n
\end{array}\right)\left(\begin{array}{c}
2 n \\
n
\end{array}\right)^{2}(n+2)(-3)^{2}+\left(\begin{array}{c}
2 n+1 \\
n+1
\end{array}\right)\left(\begin{array}{c}
2 n+2 \\
n+1
\end{array}\right)(n+3)(-3) \\
\equiv & 18\left(\begin{array}{c}
2 n \\
n
\end{array}\right)^{2}-18\left(\frac{2 n+1}{n+1}\left(\begin{array}{c}
2 n \\
n
\end{array}\right)\right)^{2} \equiv 0(\bmod n) .
\end{aligned}
$$

Therefore

$2 \sum_{k=1}^{n}(2 k+1) M_{k}^{2} \equiv 27 \sum_{k=0}^{n-1}\left(\begin{array}{c}n+1 \\ k\end{array}\right)\left(\begin{array}{c}n+k \\ k\end{array}\right)\left(\begin{array}{c}2 k \\ k\end{array}\right)(k+2)(-3)^{n-1-k}(\bmod n)$.

By (2.9) with $a=b=1$ and $q=1$, we have

$$
\sum_{k=0}^{n-1}\left(\begin{array}{c}
n+1 \\
k
\end{array}\right)\left(\begin{array}{c}
n+k \\
k
\end{array}\right)\left(\begin{array}{c}
2 k \\
k
\end{array}\right)(k+2)(-3)^{n-1-k} \equiv 0 \quad(\bmod n)
$$

Combining this with (2.11) we immediately obtain the desired (1.1).

(ii) Applying (2.7) with $n=p-1$, we get

$$
\begin{aligned}
\sum_{k=1}^{p-1}(2 k+1) M_{k}^{2}= & \sum_{k=0}^{p} \frac{(4 p-2 k-1)(p+k+1)}{p+1}\left(\begin{array}{c}
p+k \\
2 k
\end{array}\right)\left(\begin{array}{c}
2 k \\
k
\end{array}\right)\left(\begin{array}{c}
2 k+1 \\
k
\end{array}\right)(-3)^{p-k} \\
= & \sum_{k=1}^{p-1} \frac{(4 p-2 k-1)(p+k+1)}{p+1}\left(\begin{array}{c}
p \\
k
\end{array}\right)\left(\begin{array}{c}
p+k \\
k
\end{array}\right) \frac{2 k+1}{k+1}\left(\begin{array}{c}
2 k \\
k
\end{array}\right)(-3)^{p-k} \\
& +(4 p-1)(-3)^{p}+\frac{(2 p-1)(2 p+1)}{p+1}\left(\begin{array}{c}
2 p \\
p
\end{array}\right) \frac{2 p+1}{p+1}\left(\begin{array}{c}
2 p \\
p
\end{array}\right) \\
\equiv & 3 \sum_{k=1}^{p-1} \frac{p}{k}\left(\begin{array}{c}
p-1 \\
k-1
\end{array}\right)(2 k+1)^{2} \frac{\left(\begin{array}{c}
2 k \\
k
\end{array}\right)}{(-3)^{k}}+(3-12 p) 3^{p-1}-\left(2\left(\begin{array}{c}
2 p-1 \\
p-1
\end{array}\right)\right)^{2} \\
\equiv & -3 p \sum_{k=1}^{p-1}\left(4 k+4+\frac{1}{k}\right) \frac{\left(\begin{array}{c}
2 k \\
k
\end{array}\right)}{3^{k}}+3^{p}-12 p-4\left(\bmod p^{2}\right)
\end{aligned}
$$

with the aid of Wolstenholme's congruence $\left(\begin{array}{c}2 p-1 \\ p-1\end{array}\right) \equiv 1\left(\bmod p^{3}\right)(\mathrm{cf} .[\mathrm{W}])$. Com- 
bining this with (2.10) and noting that $\left(\begin{array}{c}-1 / 2 \\ k\end{array}\right)=\left(\begin{array}{c}2 k \\ k\end{array}\right) /(-4)^{k}$ for $k \in \mathbb{N}$, we obtain

$$
\begin{aligned}
-\frac{1}{12 p} \sum_{k=0}^{p-1}(2 k+1) M_{k}^{2} & \equiv 1+\sum_{k=1}^{p-1}(k+1)\left(\begin{array}{c}
-1 / 2 \\
k
\end{array}\right) \frac{(-4)^{k}}{3^{k}} \\
& \equiv \sum_{k=0}^{(p-1) / 2}\left(\begin{array}{c}
(p-1) / 2 \\
k
\end{array}\right)\left(-\frac{4}{3}\right)^{k}+\sum_{k=1}^{(p-1) / 2} k\left(\begin{array}{c}
(p-1) / 2 \\
k
\end{array}\right)\left(-\frac{4}{3}\right)^{k} \\
& \equiv\left(1-\frac{4}{3}\right)^{(p-1) / 2}-\frac{4}{3} \cdot \frac{p-1}{2} \sum_{k=1}^{(p-1) / 2}\left(\begin{array}{c}
(p-3) / 2 \\
k-1
\end{array}\right)\left(-\frac{4}{3}\right)^{k-1} \\
& \equiv\left(\frac{-3}{p}\right)+\frac{2}{3}\left(1-\frac{4}{3}\right) \\
& \equiv\left(\frac{-3}{p}\right)-2\left(\frac{-3}{p}\right)=-\left(\frac{p}{3}\right)(\bmod p) .
\end{aligned}
$$

This proves (1.2).

The proof of Theorem 1.1 is now complete.

\section{Proof of Theorem 1.2}

Lemma 3.1. Let $b, c \in \mathbb{Z}$ and $d=b^{2}-4 c$. Then

$$
b \sum_{k=0}^{n-1}(2 k+1) T_{k}(b, c)^{2}(-d)^{n-1-k}=n T_{n}(b, c) T_{n-1}(b, c) \quad \text { for any } n \in \mathbb{Z}^{+},
$$

and

$$
T_{k}(b, c)^{2}=\sum_{j=0}^{k}\left(\begin{array}{c}
k+j \\
2 j
\end{array}\right)\left(\begin{array}{c}
2 j \\
j
\end{array}\right)^{2} c^{j} d^{k-j} \quad \text { for all } k \in \mathbb{N}
$$

Remark 3.1. For (3.1) and (3.2), see [S14a, (1.19) and (4.1)].

Lemma 3.2. For any $n \in \mathbb{Z}^{+}$, we have

$$
\sum_{k=0}^{n-1} k(k+1)(8 k+9) T_{k} T_{k+1}=\frac{(-1)^{n} n}{6} \sum_{k=0}^{n-1}\left(\begin{array}{c}
n-1 \\
k
\end{array}\right)\left(\begin{array}{c}
-n-1 \\
k
\end{array}\right) C_{k} 3^{n-1-k} a(n, k)
$$

where

$a(n, k)=4 k^{2} n^{2}-8 k n^{3}-14 k^{2} n-14 k n^{2}-4 n^{3}+13 k^{2}-11 k n-26 n^{2}+39 k+4 n+26$. 
Proof. In light of (3.1) with $b=c=1$,

$$
\begin{aligned}
& \sum_{k=0}^{n-1} k(k+1)(8 k+9) T_{k} T_{k+1} \\
= & \sum_{k=1}^{n} k(k-1)(8 k+1) T_{k} T_{k-1} \\
= & \sum_{k=1}^{n}(k-1)(8 k+1) \sum_{j=0}^{k-1}(2 j+1) T_{j}^{2} 3^{k-1-j} \\
= & \sum_{j=0}^{n-1}(2 j+1) T_{j}^{2} \sum_{k=j+1}^{n}(k-1)(8 k+1) 3^{k-1-j} .
\end{aligned}
$$

By induction, for each $j \in \mathbb{N}$ we have

$$
\sum_{k=j+1}^{m}(k-1)(8 k+1) 3^{k-1-j}=\frac{1}{4}\left(3^{m-j}\left(16 m^{2}-30 m+21\right)-\left(16 j^{2}-30 j+21\right)\right)
$$

for all $m=j+1, j+2, \ldots$ Thus, in view of the above and (3.2) with $b=c=1$, we get

$$
\begin{aligned}
& 4 \sum_{k=0}^{n-1} k(k+1)(8 k+9) T_{k} T_{k+1} \\
= & \sum_{k=0}^{n}(2 k+1) T_{k}^{2}\left(3^{n-k}\left(16 n^{2}-30 n+21\right)-\left(16 k^{2}-30 k+21\right)\right)=\sum_{k=0}^{n} \sum_{l=0}^{n} F(k, l),
\end{aligned}
$$

where $F(k, l)$ denotes

$$
(2 k+1)\left(\begin{array}{c}
k+l \\
2 l
\end{array}\right)\left(\begin{array}{c}
2 l \\
l
\end{array}\right)^{2}(-3)^{k-l}\left(3^{n-k}\left(16 n^{2}-30 n+21\right)-\left(16 k^{2}-30 k+21\right)\right) .
$$

Via the telescoping method stated in [CHM, MS], the double sum can be reduced to a single sum:

$$
\sum_{k=0}^{n} \sum_{l=0}^{n} F(k, l)=\frac{2}{9} \sum_{k=0}^{n-1} \frac{a(n, k)(-3)^{n-k}(n+k) !(2 k) !}{(n-k-1) ! k !^{4}(k+1)} .
$$

Therefore

$$
\begin{aligned}
& \sum_{k=0}^{n-1} k(k+1)(8 k+9) T_{k} T_{k+1} \\
= & \frac{1}{18} \sum_{k=0}^{n-1}\left(\begin{array}{c}
n \\
k+1
\end{array}\right)\left(\begin{array}{c}
n+k \\
k
\end{array}\right)\left(\begin{array}{c}
2 k \\
k
\end{array}\right)(-3)^{n-k} a(n, k) \\
= & \frac{(-1)^{n}}{6} \sum_{k=0}^{n-1} \frac{n}{k+1}\left(\begin{array}{c}
n-1 \\
k
\end{array}\right)\left(\begin{array}{c}
-n-1 \\
k
\end{array}\right)\left(\begin{array}{c}
2 k \\
k
\end{array}\right) 3^{n-1-k} a(n, k)
\end{aligned}
$$

and hence (3.3) holds. 
Lemma 3.3. For any $n \in \mathbb{Z}^{+}$, we have

$$
n^{2}-1 \mid \sum_{k=0}^{n-1}\left(\begin{array}{c}
n-1 \\
k
\end{array}\right)\left(\begin{array}{c}
-n-1 \\
k
\end{array}\right) C_{k} 3^{n-1-k} a(n, k)
$$

with a $(n, k)$ given in Lemma 3.2.

Proof. It suffices to show that $n^{2}-1$ divides $\left(\begin{array}{c}n-1 \\ k\end{array}\right)\left(\begin{array}{c}-n-1 \\ k\end{array}\right) a(n, k)$ for any fixed $k \in\{0, \ldots, n-1\}$. Clearly,

$$
\begin{aligned}
a(n, k) & \equiv 4 k^{2}-8 k n-14 k^{2} n-14 k-4 n+13 k^{2}-11 k n-26+39 k+4 n+26 \\
& =k^{2}(17-14 n)+k(25-19 n)\left(\bmod n^{2}-1\right),
\end{aligned}
$$

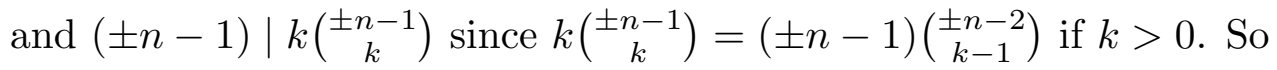

$$
\left(\begin{array}{c}
n-1 \\
k
\end{array}\right)\left(\begin{array}{c}
-n-1 \\
k
\end{array}\right) a(n, k) \equiv\left(\begin{array}{c}
n-1 \\
k
\end{array}\right)\left(\begin{array}{c}
-n-1 \\
k
\end{array}\right) k(25-19 n) \quad\left(\bmod n^{2}-1\right) .
$$

If $2 \nmid n$, then $n \pm 1$ and $25-19 n$ are all even, hence both $2(n-1)$ and $2(n+1)$ divide $\left(\begin{array}{c}n-1 \\ k\end{array}\right)\left(\begin{array}{c}-n-1 \\ k\end{array}\right) a(n, k)$. If $n$ is even, then $(n-1, n+1)=(n-1,2)=1$ and hence $n^{2}-1$ coincides with the least common multiple $[n-1, n+1]$ of $n-1$ and $n+1$. Note that when $n$ is odd we have $(2, n-1)=2$ and

$$
[2(n-1), 2(n+1)]=\frac{2(n-1) 2(n+1)}{(2(n-1), 2(n+1))}=\frac{4\left(n^{2}-1\right)}{2(n-1,2)}=n^{2}-1 .
$$

Therefore $n^{2}-1 \mid\left(\begin{array}{c}n-1 \\ k\end{array}\right)\left(\begin{array}{c}-n-1 \\ k\end{array}\right) a(n, k)$ no matter $n$ is odd or even. This concludes the proof.

Lemma 3.4. Let $a, b \in \mathbb{N}$ with $a+b$ even, and let $n \in \mathbb{Z}^{+}$. Then

$$
\left.2 n \mid \sum_{k=0}^{n-1}\left(\begin{array}{c}
n-1 \\
k
\end{array}\right)^{a}\left(\begin{array}{c}
-n-1 \\
k
\end{array}\right)\right)^{b}\left(\begin{array}{c}
2 k \\
k
\end{array}\right)(k+2) 3^{n-1-k} .
$$

Proof. Let $f(k)=k\left(\begin{array}{c}2 k-1 \\ k\end{array}\right) 3^{n-k}$ for $k=0, \ldots, n$. For each $k=0, \ldots, n-1$, we clearly have

$$
\begin{aligned}
\Delta f(k) & =f(k+1)-f(k)=(k+1)\left(\begin{array}{c}
2 k+1 \\
k+1
\end{array}\right) 3^{n-k-1}-k\left(\begin{array}{c}
2 k-1 \\
k
\end{array}\right) 3^{n-k} \\
& =(2 k+1)\left(\begin{array}{c}
2 k \\
k
\end{array}\right) 3^{n-k-1}-3 k\left(\begin{array}{c}
2 k-1 \\
k
\end{array}\right) 3^{n-1-k}=\frac{k+2}{2}\left(\begin{array}{c}
2 k \\
k
\end{array}\right) 3^{n-1-k} .
\end{aligned}
$$

Thus, by [S18a, Theorem 4.1] we get

$$
\begin{aligned}
& \sum_{k=0}^{n-1}\left(\begin{array}{c}
n-1 \\
k
\end{array}\right)^{a}\left(\begin{array}{c}
-n-1 \\
k
\end{array}\right)^{b} \frac{k+2}{2}\left(\begin{array}{c}
2 k \\
k
\end{array}\right) 3^{n-1-k} \\
= & \sum_{k=0}^{n-1}\left(\begin{array}{c}
n-1 \\
k
\end{array}\right)^{a}\left(\begin{array}{c}
-n-1 \\
k
\end{array}\right)^{b} \Delta f(k) \equiv 0(\bmod n)
\end{aligned}
$$


and hence (3.6) holds.

Proof of Theorem 1.2. Since $\left(n, n^{2}-1\right)=1$, by Lemmas 3.2 and 3.3 it suffices to show that

$$
\sum_{k=0}^{n-1}\left(\begin{array}{c}
n-1 \\
k
\end{array}\right)\left(\begin{array}{c}
-n-1 \\
k
\end{array}\right) C_{k} 3^{n-1-k} a(n, k) \equiv 0 \quad(\bmod n)
$$

For each $k=0, \ldots, n-1$, clearly

$$
a(n, k) \equiv 13 k^{2}+39 k+26=13(k+1)(k+2) \quad(\bmod n) .
$$

So

$$
\begin{aligned}
& \sum_{k=0}^{n-1}\left(\begin{array}{c}
n-1 \\
k
\end{array}\right)\left(\begin{array}{c}
-n-1 \\
k
\end{array}\right) C_{k} 3^{n-1-k} a(n, k) \\
\equiv & 13 \sum_{k=0}^{n-1}\left(\begin{array}{c}
n-1 \\
k
\end{array}\right)\left(\begin{array}{c}
-n-1 \\
k
\end{array}\right)\left(\begin{array}{c}
2 k \\
k
\end{array}\right)(k+2) 3^{n-1-k} \equiv 0(\bmod n) .
\end{aligned}
$$

with the help of Lemma 3.4. This completes the proof.

\section{Proof of Theorem 1.3}

Lemma 4.1. Let $b, c \in \mathbb{Z}$ and $d=b^{2}-4 c$. For any $n \in \mathbb{Z}^{+}$we have

$$
n T_{n}(b, c) T_{n-1}(b, c)=b \sum_{j=0}^{n-1}(n-j)\left(\begin{array}{c}
n+j \\
2 j
\end{array}\right)\left(\begin{array}{c}
2 j \\
j
\end{array}\right)^{2} c^{j} d^{n-1-j} .
$$

Proof. In view of Lemma 3.1,

$$
\begin{aligned}
n T_{n}(b, c) T_{n-1}(b, c) & =b \sum_{k=0}^{n-1}(2 k+1) \sum_{j=0}^{k}\left(\begin{array}{c}
k+j \\
2 j
\end{array}\right)\left(\begin{array}{c}
2 j \\
j
\end{array}\right)^{2} c^{j} d^{k-j}(-d)^{n-1-k} \\
& =b \sum_{j=0}^{n-1}\left(\begin{array}{c}
2 j \\
j
\end{array}\right)^{2} c^{j} d^{n-1-j} \sum_{k=j}^{n-1}(-1)^{n-1-k}(2 k+1)\left(\begin{array}{c}
k+j \\
2 j
\end{array}\right) .
\end{aligned}
$$

For each $j \in \mathbb{N}$, by induction we have

$$
\sum_{k=j}^{m-1}(-1)^{m-1-k}(2 k+1)\left(\begin{array}{c}
k+j \\
2 j
\end{array}\right)=(m-j)\left(\begin{array}{c}
m+j \\
2 j
\end{array}\right) \text { for all } m=j+1, j+2, \ldots
$$

Thus

$$
n T_{n}(b, c) T_{n-1}(b, c)=b \sum_{j=0}^{n-1}\left(\begin{array}{c}
2 j \\
j
\end{array}\right)^{2} c^{j} d^{n-1-j}(n-j)\left(\begin{array}{c}
n+j \\
2 j
\end{array}\right)
$$

and hence (4.1) holds. 
Lemma 4.2. For any $k, n \in \mathbb{Z}^{+}$with $k \leqslant n$, we have

$$
\frac{n(n+1)(n+2)}{(2, n)} \mid(n+k+1)\left(\begin{array}{c}
n+k \\
k
\end{array}\right)\left(\begin{array}{c}
n+1 \\
k+1
\end{array}\right)\left(\begin{array}{c}
2 k \\
k+1
\end{array}\right) .
$$

Proof. Clearly,

$$
\begin{aligned}
& (n+k+1)\left(\begin{array}{c}
n+k \\
k
\end{array}\right)\left(\begin{array}{l}
n+1 \\
k+1
\end{array}\right)\left(\begin{array}{c}
2 k \\
k+1
\end{array}\right) \\
= & (n+k+1)\left(\begin{array}{c}
n+k \\
k
\end{array}\right) \frac{n+1}{k+1}\left(\begin{array}{l}
n \\
k
\end{array}\right) k C_{k} \\
= & (n+1)\left(\begin{array}{c}
n+k+1 \\
k+1
\end{array}\right) n\left(\begin{array}{c}
n-1 \\
n-k
\end{array}\right) C_{k},
\end{aligned}
$$

and also

$$
(n+k+1)\left(\begin{array}{c}
n+k \\
k
\end{array}\right)\left(\begin{array}{c}
2 k \\
k+1
\end{array}\right) \equiv(k-1)(-1)^{k}\left(\begin{array}{c}
-n-1 \\
k
\end{array}\right) k C_{k} \equiv 0 \quad(\bmod n+2)
$$

since

$$
k(k-1)\left(\begin{array}{c}
-n-1 \\
k
\end{array}\right)=(-n-1)(-n-2)\left(\begin{array}{c}
-n-3 \\
k-2
\end{array}\right) \quad \text { if } k>1 .
$$

Thus

$$
[n(n+1), n+2] \mid(n+k+1)\left(\begin{array}{c}
n+k \\
k
\end{array}\right)\left(\begin{array}{c}
n+1 \\
k+1
\end{array}\right)\left(\begin{array}{c}
2 k \\
k+1
\end{array}\right)
$$

Note that

$$
[n(n+1), n+2]=\frac{n(n+1)(n+2)}{(n(n+1), n+2)}=\frac{n(n+1)(n+2)}{(2, n)} .
$$

So we have (4.3).

Lemma 4.3. For any $n \in \mathbb{N}$ we have

$$
6\left(\begin{array}{c}
2 n \\
n
\end{array}\right) \equiv 0 \quad(\bmod n+2)
$$

Proof. Observe that

$$
\left(\begin{array}{c}
2 n+2 \\
n+1
\end{array}\right)=2\left(\begin{array}{c}
2 n+1 \\
n
\end{array}\right)=\frac{2(2 n+1)}{n+1}\left(\begin{array}{c}
2 n \\
n
\end{array}\right)
$$

and hence

$$
2(2 n+1)\left(\begin{array}{c}
2 n \\
n
\end{array}\right)=(n+1)\left(\begin{array}{c}
2 n+2 \\
n+1
\end{array}\right)=(n+1)(n+2) C_{n+1} .
$$


Thus

$$
\frac{n+2}{(n+2,2 n+1)} \mid \frac{2 n+1}{(n+2,2 n+1)} 2\left(\begin{array}{c}
2 n \\
n
\end{array}\right)
$$

and hence

$$
\frac{n+2}{(n+2,2 n+1)} \mid 2\left(\begin{array}{c}
2 n \\
n
\end{array}\right) .
$$

Since $(n+2,2 n+1)=(n+2,2(n+2)-3)=(n+2,3)$ divides 3 , we obtain $(4.4)$ from (4.5).

As in $[\mathrm{S} 18 \mathrm{~b}]$, for $n \in \mathbb{Z}^{+}$we define

$$
w_{n}(x):=\sum_{k=1}^{n} w(n, k) x^{k-1} \text { with } w(n, k)=\frac{1}{k}\left(\begin{array}{l}
n-1 \\
k-1
\end{array}\right)\left(\begin{array}{l}
n+k \\
k-1
\end{array}\right) \in \mathbb{Z} .
$$

Lemma 4.4. For any integers $n \geqslant k \geqslant 1$, we have

$$
w(n, k)=\sum_{j=1}^{k}\left(\begin{array}{l}
n-j \\
k-j
\end{array}\right) N(n, j)
$$

and

$$
N(n, k)=\sum_{j=1}^{k}\left(\begin{array}{l}
n-j \\
k-j
\end{array}\right)(-1)^{k-j} w(n, j)
$$

Proof. We first prove (4.7). Observe that

$$
\begin{aligned}
\sum_{j=1}^{k}\left(\begin{array}{l}
n-j \\
k-1
\end{array}\right)(-1)^{k-j} w(n, j) & =\sum_{j=1}^{k}\left(\begin{array}{l}
n-j \\
k-j
\end{array}\right) \frac{(-1)^{k-j}}{n}\left(\begin{array}{l}
n \\
j
\end{array}\right)\left(\begin{array}{c}
n+j \\
j-1
\end{array}\right) \\
& =\frac{(-1)^{k-1}}{n}\left(\begin{array}{l}
n \\
k
\end{array}\right) \sum_{j=1}^{k}\left(\begin{array}{c}
k \\
k-j
\end{array}\right)\left(\begin{array}{c}
-n-2 \\
j-1
\end{array}\right) .
\end{aligned}
$$

Thus, with the help of the Chu-Vandermonde identity (cf. [G, (3.1)]), we get

$$
\sum_{j=1}^{k}\left(\begin{array}{l}
n-j \\
k-1
\end{array}\right)(-1)^{k-j} w(n, j)=\frac{(-1)^{k-1}}{n}\left(\begin{array}{l}
n \\
k
\end{array}\right)\left(\begin{array}{c}
k-n-2 \\
k-1
\end{array}\right)=N(n, k) .
$$

This proves (4.7).

In view of (4.7), we have

$$
\begin{aligned}
\sum_{j=1}^{k}\left(\begin{array}{l}
n-j \\
k-j
\end{array}\right) N(n, j) & =\sum_{j=1}^{k}\left(\begin{array}{l}
n-j \\
k-j
\end{array}\right) \sum_{i=1}^{j}\left(\begin{array}{l}
n-i \\
j-i
\end{array}\right)(-1)^{j-i} w(n, i) \\
& =\sum_{i=1}^{k} w(n, i)\left(\begin{array}{l}
n-i \\
k-i
\end{array}\right) \sum_{j=i}^{k}\left(\begin{array}{l}
k-i \\
j-i
\end{array}\right)(-1)^{j-i}=w(n, k) .
\end{aligned}
$$

So (4.6) also holds. This ends the proof. 
Lemma 4.5. For any $n \in \mathbb{Z}^{+}$we have

$$
w_{n}(x)=s_{n}(x)
$$

Proof. With the aid of (4.7), we get

$$
\begin{aligned}
s_{n}(x) & =\sum_{k=1}^{n} N(n, k) x^{k-1}(x+1)^{n-k} \\
& =\sum_{k=1}^{n} \sum_{j=1}^{k}\left(\begin{array}{l}
n-j \\
k-j
\end{array}\right)(-1)^{k-j} w(n, j) x^{k-1}(x+1)^{n-k} \\
& =\sum_{j=1}^{n} w(n, j) x^{n-1} \sum_{k=j}^{n}\left(\begin{array}{l}
n-j \\
k-j
\end{array}\right)(-1)^{k-j}\left(1+\frac{1}{x}\right)^{n-j-(k-j)} \\
& =\sum_{j=1}^{n} w(n, j) x^{n-1}\left(1+\frac{1}{x}-1\right)^{n-j}=w_{n}(x) .
\end{aligned}
$$

This concludes the proof.

Lemma 4.6. For any $n \in \mathbb{Z}^{+}$we have the new identity

$$
(2 x+1) \sum_{k=1}^{n} k(k+1)(2 k+1)(-1)^{n-k} w_{k}(x)^{2}=n(n+1)(n+2) w_{n}(x) w_{n+1}(x)
$$

Proof. In the case $n=1$, both sides of $(4.9)$ are equal to $6(2 x+1)$.

Now assume that (4.9) holds for a fixed positive integer $n$. Applying the Zeilberger algorithm (cf. [PWZ, pp.101-119]) via Mathematica 9 we find that

$$
(n+3) w_{n+2}(x)=(2 x+1)(2 n+3) w_{n+1}(x)-n w_{n}(x) .
$$

Thus

$$
\begin{aligned}
& (2 x+1) \sum_{k=1}^{n+1} k(k+1)(2 k+1)(-1)^{n+1-k} w_{k}(x)^{2} \\
= & (2 x+1)(n+1)(n+2)(2 n+3) w_{n+1}(x)^{2}-(2 x+1) \sum_{k=1}^{n} k(k+1)(2 k+1)(-1)^{n-k} w_{k}(x)^{2} \\
= & (2 x+1)(n+1)(n+2)(2 n+3) w_{n+1}(x)^{2}-n(n+1)(n+2) w_{n}(x) w_{n+1}(x) \\
= & (n+1)(n+2) w_{n+1}(x)\left((2 x+1)(2 n+3) w_{n+1}(x)-n w_{n}(x)\right) \\
= & (n+1)(n+2)(n+3) w_{n+1}(x) w_{n+2}(x) .
\end{aligned}
$$

In view of the above, by induction, (4.9) holds for each $n \in \mathbb{Z}^{+}$. 
Proof of Theorem 1.3. (i) Let $\delta \in\{0,1\}$. In light of Lemma 4.1,

$$
\begin{aligned}
& \sum_{k=1}^{n} k^{2 \delta+1} T_{k}(b, c) T_{k-1}(b, c) d^{n-k} \\
= & \sum_{k=1}^{n} k^{2 \delta} b \sum_{j=0}^{k-1}(k-j)\left(\begin{array}{c}
k+j \\
2 j
\end{array}\right)\left(\begin{array}{c}
2 j \\
j
\end{array}\right)^{2} c^{j} d^{k-1-j} d^{n-k} \\
= & b \sum_{j=0}^{n-1}\left(\begin{array}{c}
2 j \\
j
\end{array}\right)^{2} c^{j} d^{n-1-j} \sum_{k=j+1}^{n} k^{2 \delta}(k-j)\left(\begin{array}{c}
k+j \\
2 j
\end{array}\right) .
\end{aligned}
$$

By induction, for each $j \in \mathbb{N}$, we have

$$
\sum_{k=j+1}^{m} k^{2 \delta}(k-j)\left(\begin{array}{c}
k+j \\
2 j
\end{array}\right)=\frac{m^{\delta}(m+1)^{\delta}}{2} \cdot \frac{(m-j)(m+j+1)}{j+\delta+1}\left(\begin{array}{c}
m+j \\
2 j
\end{array}\right)
$$

for every $m=j+1, j+2, \ldots$ Therefore,

$$
\begin{aligned}
& \sum_{k=1}^{n} k^{2 \delta+1} T_{k}(b, c) T_{k-1}(b, c) d^{n-k} \\
= & b \frac{n^{\delta}(n+1)^{\delta}}{2} \sum_{j=0}^{n-1}\left(\begin{array}{c}
2 j \\
j
\end{array}\right)^{2} c^{j} d^{n-1-j} \frac{(n-j)(n+j+1)}{j+\delta+1}\left(\begin{array}{c}
n+j \\
2 j
\end{array}\right) \\
= & \frac{b}{2}(n(n+1))^{\delta} \sum_{j=0}^{n-1} \frac{\left(\begin{array}{c}
2 j \\
j
\end{array}\right)}{j+\delta+1} c^{j} d^{n-1-j}(n-j)(n+j+1)\left(\begin{array}{c}
n \\
j
\end{array}\right)\left(\begin{array}{c}
n+j \\
j
\end{array}\right)
\end{aligned}
$$

and hence

$$
\begin{aligned}
& \sum_{k=1}^{n} k^{2 \delta+1} T_{k}(b, c) T_{k-1}(b, c) d^{n-k} \\
= & \frac{b}{2}(n(n+1))^{\delta+1} \sum_{j=0}^{n-1}\left(\begin{array}{c}
n-1 \\
j
\end{array}\right)\left(\begin{array}{c}
n+j+1 \\
j
\end{array}\right) \frac{\left(\begin{array}{c}
2 j \\
j
\end{array}\right)}{j+\delta+1} c^{j} d^{n-1-j} .
\end{aligned}
$$

In the case $\delta=0,(4.11)$ yields (1.4) since $\left(\begin{array}{c}2 j \\ j\end{array}\right) /(j+1)=C_{j} \in \mathbb{Z}$. By Lemma 4.3 and (4.11) with $\delta=1$, we immediately obtain (1.5).

(ii) By induction, for each $j \in \mathbb{N}$ we have

$$
\sum_{k=j}^{m}(2 k+1)\left(\begin{array}{c}
k+j \\
2 j
\end{array}\right)=\frac{(m+1)(m+j+1)}{j+1}\left(\begin{array}{c}
m+j \\
2 j
\end{array}\right) \quad \text { for all } m=j, j+1, \ldots
$$


In view of this and (2.4), we have

$$
\begin{aligned}
& \sum_{k=1}^{n} k(k+1)(2 k+1) s_{k}(x)^{2} \\
= & \sum_{k=1}^{n}(2 k+1) \sum_{j=1}^{k}\left(\begin{array}{c}
k+j \\
2 j
\end{array}\right)\left(\begin{array}{c}
2 j \\
j
\end{array}\right)\left(\begin{array}{c}
2 j \\
j+1
\end{array}\right)(x(x+1))^{j-1} \\
= & \sum_{j=1}^{n}\left(\begin{array}{c}
2 j \\
j
\end{array}\right)\left(\begin{array}{c}
2 j \\
j+1
\end{array}\right)(x(x+1))^{j-1} \sum_{k=j}^{n}(2 k+1)\left(\begin{array}{c}
k+j \\
2 j
\end{array}\right) \\
= & \sum_{j=1}^{n}\left(\begin{array}{c}
2 j \\
j
\end{array}\right)\left(\begin{array}{c}
2 j \\
j+1
\end{array}\right)(x(x+1))^{j-1} \frac{(n+1)(n+j+1)}{j+1}\left(\begin{array}{c}
n+j \\
2 j
\end{array}\right) \\
= & \sum_{j=1}^{n}\left(\begin{array}{c}
2 j \\
j+1
\end{array}\right)(x(x+1))^{j-1} \frac{(n+1)(n+j+1)}{j+1}\left(\begin{array}{c}
n \\
j
\end{array}\right)\left(\begin{array}{c}
n+j \\
j
\end{array}\right)
\end{aligned}
$$

and hence

$$
\sum_{k=1}^{n} k(k+1)(2 k+1) s_{k}(x)^{2}=\sum_{k=1}^{n}(n+k+1)\left(\begin{array}{c}
n+1 \\
k+1
\end{array}\right)\left(\begin{array}{c}
n+k \\
k
\end{array}\right)\left(\begin{array}{c}
2 k \\
k+1
\end{array}\right)(x(x+1))^{k-1} \text {. }
$$

Let $x=(b / \sqrt{d}-1) / 2$. Then $x(x+1)=c / d$. In view of Lemma 2.1(ii) and (4.13), we have

$$
\begin{aligned}
& \sum_{k=0}^{n-1}(k+1)(k+2)(2 k+3) M_{k}(b, c)^{2} d^{n-1-k} \\
= & \sum_{k=0}^{n-1}(k+1)(k+2)(2 k+3) d^{k} s_{k+1}(x)^{2} d^{n-1-k} \\
= & d^{n-1} \sum_{k=1}^{n} k(k+1)(2 k+1) s_{k}(x)^{2} \\
= & \sum_{k=1}^{n}(n+k+1)\left(\begin{array}{c}
n+1 \\
k+1
\end{array}\right)\left(\begin{array}{c}
n+k \\
k
\end{array}\right)\left(\begin{array}{c}
2 k \\
k+1
\end{array}\right) c^{k-1} d^{n-k} .
\end{aligned}
$$

Combining this with Lemma 4.2, we get the desired (1.6). 
In light of Lemma 2.1(ii) and Lemmas 4.5-4.6, we have

$$
\begin{aligned}
& \sum_{k=0}^{n-1}(k+1)(k+2)(2 k+3) M_{k}(b, c)^{2}(-d)^{n-1-k} \\
= & \sum_{k=0}^{n-1}(k+1)(k+2)(2 k+3) d^{k} s_{k+1}(x)^{2}(-d)^{n-1-k} \\
= & d^{n-1} \sum_{k=1}^{n} k(k+1)(2 k+1)(-1)^{n-k} w_{k}(x)^{2} \\
= & n(n+1)(n+2) d^{n-1} \frac{s_{n}(x) s_{n+1}(x)}{2 x+1} \\
= & n(n+1)(n+2) d^{n-1} \frac{M_{n-1}(b, c)}{\sqrt{d}^{n-1}} \cdot \frac{M_{n}(b, c)}{\sqrt{d}^{n}} \cdot \frac{\sqrt{d}}{b} \\
= & n(n+1)(n+2) \frac{M_{n}(b, c) M_{n-1}(b, c)}{b} .
\end{aligned}
$$

If $2 \nmid n$ then $b \mid M_{n}(b, c)$; if $2 \mid n$ then $2 \nmid n-1$ and $b \mid M_{n-1}(b, c)$. So $b$ divides $M_{n}(b, c) M_{n-1}(b, c)$. Therefore (1.7) holds.

The proof of Theorem 1.3 is now complete.

\section{Some open PROBlems}

Clearly,

$$
\frac{\left(\begin{array}{c}
2 k \\
k
\end{array}\right)}{2 k-1}=\frac{2}{2 k-1}\left(\begin{array}{c}
2 k-1 \\
k
\end{array}\right)=\frac{2}{k}\left(\begin{array}{c}
2 k-2 \\
k-1
\end{array}\right)=2 C_{k-1} \text { for } k \in \mathbb{Z}^{+},
$$

and thus $2 k-1 \mid\left(\begin{array}{c}2 k \\ k\end{array}\right)$ for all $k \in \mathbb{N}$. Motivated by this we introduce a new kind of numbers

$$
W_{n}:=\sum_{k=0}^{\lfloor n / 2\rfloor}\left(\begin{array}{c}
n \\
2 k
\end{array}\right) \frac{\left(\begin{array}{c}
2 k \\
k
\end{array}\right)}{2 k-1} \quad(n=0,1,2, \ldots)
$$

which are analogues of the Motzkin numbers. The values of $W_{0}, W_{1}, \ldots, W_{12}$ are as follows:

$$
-1,-1,1,5,13,29,63,139,317,749,1827,4575,11699 .
$$

Applying the Zeilberger algorithm (cf. [PWZ, pp.101-119]) via Mathematica 9, we obtain the recurrence

$$
(n+3) W_{n+3}=(3 n+7) W_{n+2}+(n-5) W_{n+1}-3(n+1) W_{n}(n=0,1,2, \ldots) .
$$

For this new kind of numbers, we have the following conjecture similar to Theorem 1.1. 
Conjecture 5.1. (i) For any $n \in \mathbb{Z}^{+}$we have

$$
\sum_{k=0}^{n-1}(8 k+9) W_{k}^{2} \equiv n \quad(\bmod 2 n) .
$$

Also, for any odd prime $p$ we have

$$
\frac{1}{p} \sum_{k=0}^{p-1}(8 k+9) W_{k}^{2} \equiv 24+10\left(\frac{-1}{p}\right)-9\left(\frac{p}{3}\right)-18\left(\frac{3}{p}\right) \quad(\bmod p) .
$$

(ii) For any prime $p>3$ and positive integer $n$, the number

$$
\frac{\sum_{k=0}^{p n-1} W_{k}^{2}-2\left(\sum_{k=0}^{n-1} T_{k}\right)^{2}}{p n}
$$

is always a p-adic integer.

Remark 5.1. We also guess that the sequence $\left(W_{n+1} / W_{n}\right)_{n \geqslant 5}$ is strictly increasing to the limit 3 and the sequence $\left(\sqrt[n+1]{W_{n+1}} / \sqrt[n]{W_{n}}\right)_{n \geqslant 9}$ is strictly decreasing to the limit 1.

For $h, n \in \mathbb{Z}^{+}$, we define

$$
w_{n}^{(h)}(x):=\sum_{k=1}^{n} w(n, k)^{h} x^{k-1} .
$$

Conjecture 5.2. Let $h, m, n \in \mathbb{Z}^{+}$. Then

$$
\frac{(2, n)}{n(n+1)(n+2)} \sum_{k=1}^{n} k(k+1)(2 k+1) w_{k}^{(h)}(x)^{m} \in \mathbb{Z}[x] .
$$

Also,

$$
\frac{(2, m-1, n)}{n(n+1)(n+2)} \sum_{k=1}^{n}(-1)^{k} k(k+1)(2 k+1) w_{k}(x)^{m} \in \mathbb{Z}[x]
$$

and

$$
\frac{1}{n(n+1)(n+2)} \sum_{k=1}^{n}(-1)^{k} k(k+1)(2 k+1) w_{k}^{(h)}(x)^{m} \in \mathbb{Z}[x] \text { for } h>1 .
$$

Remark 5.2. Fix $n \in \mathbb{Z}^{+}$. By combining (4.13) with Lemma 4.2, we obtain

$$
\frac{(2, n)}{n(n+1)(n+2)} \sum_{k=1}^{n} k(k+1)(2 k+1) s_{k}(x)^{2} \in \mathbb{Z}[x(x+1)] .
$$

As $s_{k}(x)=w_{k}(x)$ for all $k \in \mathbb{Z}^{+}$(by Lemma 4.5), this implies (5.4) with $h=1$ and $m=2$. Since $w_{2 j}(x) /(2 x+1) \in \mathbb{Z}[x]$ for all $j \in \mathbb{Z}^{+}$(cf. [S18b, Section 4]), (5.5) with $m=2$ follows from (4.9).

For $h \in \mathbb{Z}^{+}$and $n \in \mathbb{N}$, we define

$$
D_{n}^{(h)}(x):=\sum_{k=0}^{n}\left(\begin{array}{c}
n \\
k
\end{array}\right)^{h}\left(\begin{array}{c}
n+k \\
k
\end{array}\right)^{h} x^{k} \text { and } S_{n}^{(h)}(x):=\sum_{k=0}^{n}\left(\begin{array}{c}
n+k \\
2 k
\end{array}\right)^{h} C_{k}^{h} x^{k} .
$$

Note that $S_{n}^{(1)}(x)=S_{n}(x)$ for all $n \in \mathbb{N}$. 
Conjecture 5.3. Let $h, m, n \in \mathbb{Z}^{+}$.

(i) We have

$$
\frac{(2, n)}{n(n+1)(n+2)} \sum_{k=1}^{n} k(k+1)(2 k+1) S_{k}^{(h)}(x)^{m} \in \mathbb{Z}[x]
$$

and

$$
\frac{(2, m-1, n)}{n(n+1)(n+2)} \sum_{k=1}^{n}(-1)^{k} k(k+1)(2 k+1) S_{k}^{(h)}(x)^{m} \in \mathbb{Z}[x] .
$$

(ii) We have

$$
\frac{(2, n)}{n(n+1)(n+2)} \sum_{k=1}^{n} k(k+1)(2 k+1) D_{k}^{(h)}(x)^{m} \in \mathbb{Z}[x]
$$

and

$$
\frac{(2, h m-1, n)}{n(n+1)(n+2)} \sum_{k=1}^{n}(-1)^{k} k(k+1)(2 k+1) D_{k}^{(h)}(x)^{m} \in \mathbb{Z}[x] .
$$

Remark 5.3. Fix $n \in \mathbb{Z}^{+}$. As $S_{k}(x)=(x+1) s_{k}(x)=(x+1) w_{k}(x)$ for all $k \in \mathbb{Z}^{+}$, (5.8) and (5.9) with $h=1$ and $m=2$ do hold in view of Remark 5.2. We also conjecture that

$$
\frac{2}{3 n(n+1)} \sum_{k=1}^{n}(-1)^{n-k} k^{2} D_{k} D_{k-1} \text { and } \frac{1}{n} \sum_{k=1}^{n}(-1)^{n-k}\left(4 k^{2}+2 k-1\right) D_{k-1} s_{k}
$$

are positive odd integers.

Conjecture 5.4. (i) For any $h, m, n \in \mathbb{Z}^{+}$we have

$$
\frac{2(2, n)}{n(n+1)(n+2)} \sum_{k=1}^{n} k(k+1)(k+2)\left(w_{k}^{(h)}(x) w_{k+1}^{(h)}(x)\right)^{m} \in \mathbb{Z}[x]
$$

(ii) For any $m, n \in \mathbb{Z}^{+}$we have

$$
\frac{2(2, n)}{n(n+1)(n+2)(2 x+1)^{m}} \sum_{k=1}^{n} k(k+1)(k+2)\left(w_{k}(x) w_{k+1}(x)\right)^{m} \in \mathbb{Z}[x] .
$$

If $n \in \mathbb{Z}^{+}$is even, then

$$
\frac{4}{n(n+1)(n+2)(2 x+1)^{3}} \sum_{k=1}^{n} k(k+1)(k+2) w_{k}(x) w_{k+1}(x) \in \mathbb{Z}[x] .
$$

Remark 5.4. Recall that $w_{2 j}(x) /(2 x+1) \in \mathbb{Z}[x]$ for all $j \in \mathbb{Z}^{+}$(by [S18b, Section $4])$.

Acknowledgments. The author would like to thank Prof. Qing-Hu Hou and the anonymous referee for their helpful comments. 


\section{REFERENCES}

[CHM] W. Y. C. Chen, Q.-H. Hou, and Y.-P. Mu, A telescoping method for double summations, J. Comput. Appl. Math. 196 (2006), 553-566.

[G] H. W. Gould, Combinatorial Identities, Morgantown Printing and Binding Co., 1972.

[Gr] R. P. Grimaldi, Fibonacci Numbers and Catalan Numbers: An Introduction, John Wiley \& Sons, New Jersey, 2012.

[MS] Y.-P. Mu and Z.-W. Sun, Telescoping method and congruences for double sums, Int. J. Number Theory 14 (2018), 143-165.

[O] G. Olive, Generalized powers, Amer. Math. Monthly 72 (1965), 619-627.

[PWZ] M. Petkovšek, H. S. Wilf and D. Zeilberger, $A=B$, A K Peters, Wellesley, 1996.

[St97] R. P. Stanley, Hipparchus, Plutarch, Schröder, and Hough, Amer. Math. Monthly 104 (1997), 344-350.

[St99] R. P. Stanley, Enumerative Combinatorics, Vol. 2, Cambridge Univ. Press, Cambridge, 1999.

[S11] Z.-W. Sun, On Delannoy numbers and Schröder numbers, J. Number Theory 131 (2011), $2387-2397$.

[S12a] Z.-W. Sun, On sums involving products of three binomial coefficients, Acta Arith. 156 (2012), 123-141.

[S12b] Z.-W. Sun, On sums of binomial coefficients modulo $p^{2}$, Colloq. Math. 127 (2012), 39-54.

[S14a] Z.-W. Sun, Congruences involving generalized central trinomial coefficients, Sci. China Math. 57 (2014), 1375-1400.

[S14b] Z.-W. Sun, On sums related to central binomial and trinomial coefficients, in: M. B. Nathanson (ed.), Combinatorial and Additive Number Theory: CANT 2011 and 2012, Springer Proc. in Math. \& Stat., Vol. 101, Springer, New York, 2014, pp. 257-312.

[S18a] Z.-W. Sun, Two new kinds of numbers and related divisibility results, Colloq. Math. 154 (2018), 241-273.

[S18b] Z.-W. Sun, Arithmetic properties of Delannoy numbers and Schröder numbers, J. Number Theory 183 (2018), 146-171.

[S20] Z.-W. Sun, New series for powers of $\pi$ and related congruences, Electron. Res. Arch. 28 (2020), 1273-1342.

[W] J. Wolstenholme, On certain properties of prime numbers, Quart. J. Appl. Math. 5 (1862), $35-39$. 